\title{
GUAMAN POMA Y EL REPERTORIO ANÓNIMO (1554): UNA NUEVA FUENTE PARA LAS EDADES DEL MUNDO EN LA NUEVA CORÓNICA Y BUEN GOBIERNO
}

\author{
GUAMAN POMA AND THE ANONYMOUS REPERTORIO (1554): \\ A NEW SOURCE FOR AGES OF THE WORLD IN THE NUEVA CORÓNICA \\ Y BUEN GOBIERNO
}

\author{
Soledad González Díaz ${ }^{1}$
}

\begin{abstract}
El presente estudio parte de una lectura atenta de las descripciones de los linajes bíblicos presentes en las tres primeras edades del mundo de la Nueva Corónica. Dado que éstos presentan errores de sucesión que no existen ni en el Antiguo Testamento ni en la Chronographia de Hieronimo de Chaves -texto que Guaman Poma utilizó como modelo para su capítulo de las edades del mundose propone que el cronista contó con una fuente alternativa en la redacción de este apartado: un Repertorio de los Tiempos escrito por un autor anónimo e impreso en Valladolid en 1554. A partir de la filiación de ambos textos y de la Chronographia de Chaves se plantea que Guaman Poma intentó elaborar una representación lineal y coherente del pasado occidental y andino, asumiendo que las edades del mundo y las edades andinas habían tenido lugar en forma simultánea y adoptando un esquema temporal de cinco eras para cada una de ellas.
\end{abstract}

Palabras claves: Guaman Poma, Chronographia o Repertorio de Tiempos, Repertorio Anónimo, Antiguo Testamento.

This study starts from a careful reading of the descriptions of biblical lineages in the first three ages of the world in the Nueva Corónica. Because there are errors of succession in them that do not exist in the Old Testament or in Hieronimo de Chaves' Chronographia-the text that Guaman Poma used as a model for his chapter on the ages of the world-it is proposed that the chronicler had an alternative source in writing this section, a Repertorio de los Tiempos written by an anonymous author and printed in Valladolid in 1554. From the affiliation of both texts and Chaves' Chronographia, it is argued that Guaman Poma attempted to develop a linear and coherent representation of both the western and the Andean past, assuming that the ages of the world and Andean ages had taken place simultaneously and taking a temporal scheme of five eras for each one of them.

Key words: Guaman Poma, Chronographia o Repertorio de Tiempos, Repertorio Anónimo, Old Testament.

La periodización del pasado que Guaman Poma elaboró en su Nueva Corónica y Buen Gobierno consiste en dos series paralelas de cinco edades, una del mundo y otra de los indios, desarrolladas independientemente a lo largo de cuatro capítulos. La principal diferencia entre ambas series radica en que mientras las edades del mundo dan cuenta de un pasado común para toda la humanidad, inspirado en la tradición patrística y medieval (Tabla 1), las edades de los indios corresponden a una periodización exclusiva para el pasado andino, que Guaman Poma dividió en tiempos de Vari Vira Cocha Runa, Vari Runa, Purun Runa, Auca Runa e Inca Runa (2004 [1615/1616]:48-119).

Desde el hallazgo de la crónica a principios del siglo XX, las edades del mundo y de los indios han sido objeto de diversos estudios centrados en tratar de establecer la procedencia y la eventual relación entre ambas series, con el objetivo de determinar si detrás de las edades de los indios subyacían referentes europeos o si, por el contrario, se trataba de una tradición netamente andina. Dado que hacia la misma época el arqueólogo Julio Tello y sus colaboradores descubrieron las antiguas civilizaciones Chavín y Paracas, la posibilidad de pensar el pasado andino en términos mucho más extensos que el período Inca ya no parecía imposible. Por ende, las periodizaciones de la Nueva Corónica se presentaban como una alternativa plausible en la búsqueda de un correlato textual a las evidencias arqueológicas que daban cuenta de ese pasado, no obstante los errores factuales y cronológicos que

1 Programa de Doctorado en Filología Española de la Universidad Autónoma de Barcelona, Barcelona, España, y Programa de Capital Humano Avanzado CONICYT, Santiago. Chile. horrorflorido@gmail.com 
Tabla 1. Las edades del mundo en Hieronimo de Chaves, Guaman Poma y el Repertorio anónimo.

The ages of the world in Hieronimo de Chaves, Guaman Poma and the anonymous Repertorio.

\begin{tabular}{lccc}
\hline & Chaves & Guaman Poma & Anónimo \\
& $(1584[1548]: 57 \mathrm{v}-64)$ & $(2004[1615 / 1616]: 22-47)$ & $(1554: 71-72)$ \\
\hline $1^{\mathrm{a}}$ edad & Adán - Noé & Adán - Noé & Adán - Noé \\
$2^{\mathrm{a}}$ edad & Noé - Abraham & Noé - Abraham & Noé - Abraham \\
$3^{\mathrm{a}}$ edad & Abraham - David & Abraham - David & Abraham - Moisés \\
$4^{\mathrm{a}}$ edad & David - Cyro & David - Jesucristo & Moisés - David \\
$5^{\text {a edad }}$ & Cyro - Jesucristo & Jesucristo & David - Jesucristo \\
$6^{\text {a edad }}$ & Jesucristo - Juicio final & - & Jesucristo - Juicio Final \\
$7^{\text {a edad }}$ & - & - & Juicio Final \\
\hline
\end{tabular}

investigadores como Raúl Porras evidenciaron a la hora de aproximarse críticamente a la obra de Guaman Poma (Duviols 1980).

En la década de los setenta, los errores cronológicos que Porras identificó en la Nueva Corónica fueron analizados bajo una nueva óptica por Nathan Wachtel (1976:249-251) que, recordando que el cronista escribía desde su propio presente, proponía que Guaman Poma había utilizado la cronología occidental para ajustar su narración a un esquema temporal de cinco edades, sugiriendo que podría tratarse de una antigua noción indígena de cinco edades de mil años cada una. En la misma línea, Juan Ossio (1973) planteaba que las categorías con las que Guaman Poma había pensado su pasado eran distintas de aquellas que había utilizado para expresarse, puesto que si bien en su forma de escribir el cronista seguía la estructura y el orden propios de la historiografía europea de su tiempo, detrás de ese ropaje extranjero emergían categorías míticas que conllevaban una concepción estática del tiempo y una visión unitaria del cosmos y de las relaciones sociales.

A fines de la década de los setenta y desde una perspectiva más sincrónica, Rolena Adorno (1978) propuso una lectura de la Nueva Corónica en función a las fuentes que el propio Guaman Poma había utilizado en su proceso de redacción, más allá de los capítulos de las edades del mundo y de los indios. Adorno determinó que el cronista no siempre explicitaba sus fuentes a la hora de escribir, evidenciando las similitudes entre la Nueva Corónica y textos como el Tratado de las Doce Dudas de Bartolomé de las Casas y los Avisos para Confesores de Jerónimo de Loaysa, a quienes Guaman Poma había seguido en su defensa de la legitimidad de los títulos de los indios sobre sus tierras, sin citarlos. Otra fuente no explícita de la Nueva Corónica había sido la Instrucción para descubrir todas las guacas del Perú de Cristóbal de Albornoz, para quien Guaman Poma había trabajado en la campaña de extirpación del movimiento del Taki Unquy en la provincia de Lucanas, entre 1566 y 1570 . Adorno también evidenció la relación entre la Nueva Corónica y textos como el Tercero Cathecismo, cuya prosa "estridente y amenazante" fue adoptada por Guaman Poma como modelo de escritura, y el Symbolo Cathólico Indiano del franciscano Gerónimo de Oré, cuya familia fue cercana a la del cronista y a quien éste convirtió en su verdadero "héroe literario".

A principios de la década de los ochenta, Pierre Duviols (1980) constataba que el modelo histórico de Guaman Poma era lineal y finito y que seguía los cánones de representación de la historia renacentista basados en la reinterpretación de las fuentes grecolatinas y medievales como Hesíodo, Tertuliano, Lactancio, San Agustín y Santo Tomás. El autor afirmaba que el capítulo de las edades de los indios no se originaba en la tradición oral de los Andes -a pesar de los nombres quechuas que designaban las cinco edades-, sino en una filosofía de la historia que descansaba sobre la idea de que el progreso material de las sociedades andinas había implicado la paulatina regresión moral de sus costumbres. Poco después y retomando su trabajo anterior, Duviols (1983) planteaba que el capítulo de las edades de los indios había sido elaborado por una mente hábil y conocedora de los argumentos teológicos y jurídicos de la España colonizadora, teniendo como modelo los cuatro imperios de las profecías de Daniel 7. Esta mente hábil había sido la de Francisco Fernández de Córdova, un criollo licenciado en derecho en la Universidad de Lima y 
perteneciente a una de las familias más ilustres del virreinato, autor de unos cuadernos hoy desaparecidos que habrían contenido, de acuerdo a Duviols, la idea original de las edades de los indios y a los cuales Guaman Poma habría tenido acceso en Huamanga. De paso, la existencia de estos cuadernos explicaba las evidentes similitudes entre la Nueva Corónica y el Memorial de las Historias del Nuevo Mundo de Fray Buenaventura de Salinas y Córdova, que también habría obtenido la periodización de las edades de los indios de dicha fuente. Respondiendo a Duviols y reivindicando la autoría de Guaman Poma, Jean-Philippe Husson (1995) determinó que los cuadernos perdidos de Fernández de Córdova -en caso de existir- no habían constituido una fuente común para el cronista y Salinas y que el parecido entre ambos se debía a que este último había copiado de la Nueva Corónica las edades de los indios, interpretando de manera muy personal sus dibujos y suponiendo que los títulos y las palabras que aparecían en ellos correspondían a nombres de individuos y no al de comunidades humanas, como en efecto lo eran. Husson también planteaba que tras el barniz ideológico cristiano de Guaman Poma se ocultaban las huellas de diferentes tradiciones prehispánicas, tanto cusqueñas como ayacuchanas, reflejadas sobre todo en las diferencias dialectales del quechua utilizadas por el cronista.

Las investigaciones sobre las periodizaciones utilizadas por Guaman Poma dieron un nuevo giro cuando Mónica Barnes (1995) y David Fleming (1994) -de una parte- y Sophie Plas (1996) -de otra- demostraron que una de las fuentes que Guaman Poma había utilizado en la redacción de las edades del mundo había sido la Chronographia o Reportorio de los Tiempos del cosmógrafo Hieronimo de Chaves (Adorno 2001). Aunque sin hacer referencia explícita a la obra, tal como había sucedido con el Tratado de las Doce Dudas, Guaman Poma siguió la Chronographia en el capítulo de las edades del mundo y en el de los reinados de los papas, copiando los extensos listados de patriarcas bíblicos, reyes de la antigüedad y papas en torno a los cuales Chaves, siguiendo la tradición de los cronicones medievales, había construido sus tablas cronológicas. Guaman Poma también siguió la Chronographia en el segundo capítulo de los meses del año (2004:1140-1178 [1615/1616]), cuestión que por razones de espacio no abordaremos en el presente artículo.
La Chronographia fue publicada por primera vez en Sevilla en 1548 y desde entonces hasta 1588 se realizaron quince ediciones diferentes, convirtiéndose en uno de los libros más vendidos de la época ${ }^{1}$. Ejemplares de ella fueron inventariados en las bibliotecas del Inca Garcilaso (Durand 1948:251), del famoso extirpador de idolatrías Francisco de Ávila (Hampe 1996a:135) y de Juan Hurtado de Vera, canónigo de la iglesia metropolitana de Lima (Hampe 1996b:178), lo que evidencia que este libro también circuló ampliamente en el Perú. Su autor, Hieronimo de Chaves, fue astrólogo, cosmógrafo y Piloto Mayor de la Casa de Contratación de Sevilla y tiempo antes de escribir la Chronographia había realizado la traducción del latín al español del Tractatus de Sphaera Mundi de Johannes de Sacrobosco, un importante estudio de astronomía medieval inspirado en el modelo ptolemaico.

No obstante las similitudes conceptuales, formales y textuales que Sophie Plas (1996) encontró entre la Chronographia y la Nueva Corónica, ambos registros diferían en el número de edades que asignaban al pasado occidental, en la medida en que las edades del mundo en la Chronographia no eran cinco como en la Nueva Corónica, sino seis (Tabla 1). Dada esta diferencia, Plas (1996:112) sugirió que era posible que las cinco edades de Guaman Poma estuviesen inspiradas en un modelo europeo distinto al de Chaves y aún no determinado, mientras que Fleming (1994:52) propuso que esta diferencia se debía a que Guaman Poma había intentado acomodar el mundo andino a un contexto cronológico europeo y cristiano, que descansaba sobre el supuesto de que el imperio Inca y la era cristiana comenzaban al mismo tiempo.

El punto de partida del presente estudio se originó en una lectura atenta de las descripciones de las genealogías bíblicas presentes en las tres primeras edades del mundo de la Nueva Corónica, en las que Guaman Poma reiteradamente confunde las descendencias de los patriarcas del Antiguo Testamento. Dado que en las tablas cronológicas de Chaves esta sucesión es correcta y que el autor no hace mención alguna a los detalles de las vidas de los patriarcas, la hipótesis inicial fue que Guaman Poma debía haber utilizado una fuente alternativa para la redacción del capítulo de las edades del mundo, de la cual había extraído la información con la que "completó" las genealogías de Chaves. En consecuencia, los errores o bien ya estaban presentes en esa fuente desconocida o, en su defecto, 
habían sido introducidos por el propio autor en el proceso de copia de ésta. Creemos que esta fuente desconocida fue un Repertorio de los Tiempos distinto al de Hieronimo de Chaves, de un autor anónimo e impreso en Valladolid en $1554^{2}$.

\section{El Repertorio Anónimo, Hieronimo de Chaves y Guaman Poma}

El Repertorio de los Tiempos al que hemos hecho referencia anteriormente fue publicado en la casa de Francisco Fernández de Córdova -no confundir con su homónimo limeño mencionado más arriba- por un autor desconocido que se identificó en la portada como "un religioso de la horden del glorioso doctor sant Bernardo". El libro no posee ningún tipo de licencia de impresión, privilegio de venta o provisión real y gran parte de sus capítulos son idénticos a los de la Chronographia de Chaves, así como también el título ${ }^{3}$. Dado que la primera edición de esta última es seis años anterior a la fecha de publicación del Anónimo, parece bastante probable que éste corresponda a una edición pirata del texto de Chaves, adaptada por su autor desconocido para un público menos erudito que el del cosmógrafo, en la medida en que todos aquellos capítulos de la Chronographia que implicaban un mayor grado de conocimiento astronómico y matemático no fueron incluidos en él ${ }^{4}$. No obstante las similitudes, el Anónimo también innovó en determinadas temáticas, una de las cuales precisamente fue la de las edades del mundo. Chaves había organizado sus tablas en torno a un criterio estrictamente cronológico, siguiendo el canon que los Padres de la Iglesia y los cronicones medievales habían utilizado para calcular el Anno Mundi o edad del mundo, que partía con el linaje de Adán y continuaba por la línea sucesoria de su hijo Set hasta terminar con Jesucristo. Los comentarios de Chaves a los personajes que componían esta sucesión fueron casi nulos, remitiéndose sólo a las diferentes duraciones que los calculistas habían asignado a cada edad, distinguiendo entre la cronología de la Vulgata, la Septuaginta, Alfonso X y Beda el Venerable. El Anónimo, por el contrario, no incluyó ninguna tabla cronológica en su descripción de las edades del mundo, esbozando sólo una pequeña síntesis de cada época con episodios asociados a las vidas de los patriarcas y reyes de la Antigüedad, incluso si éstos no contaban para el cálculo del Anno Mundi. Esta síntesis no fue en absoluto convencional, pues su autor desconocido rescató los hechos más escabrosos de estos personajes, con una preocupación poco usual por los números y las cantidades asociados a ellos. Así, por ejemplo, en su descripción de la cuarta edad menciona a "Sangar que mato seyscientos hombres con vna reja y la muger Jahel que mato al capitan Sifara, metiendole vn clavo por las sienes y Samson que mato mil hombres con vna quixada de asno" (Anónimo 1554:71v). Otra característica particular del Anónimo fue que al integrar más personajes a su descripción, no distinguió cuáles pertenecían o no a la descendencia de Set que había seguido Chaves y que constituía el eje de la narración de Guaman Poma. Esta cuestión es fundamental si se tiene en cuenta que en el libro del Génesis existen personajes homónimos o que, en su defecto, poseen nombres muy similares, a pesar de pertenecer a líneas sucesorias distintas. Otra diferencia entre Chaves y el Anónimo es que cada uno utilizó una periodización distinta para las edades del mundo, ya que en este último la cuarta edad comienza con Moisés, la quinta con David, y el rey Ciro no marca el inicio de ninguna edad (Tabla 1).

Como veremos a continuación, Guaman Poma al redactar el capítulo correspondiente a las edades del mundo combinó ambos registros, completando las genealogías de Chaves con la información del Anónimo, al mismo tiempo que agregaba sus propias reflexiones sobre el pasado occidental. Sin embargo, en el proceso de compatibilizar los dos repertorios - de un lado-y su conocimiento personal -de otro-, el cronista agregó a la línea de Set que copió de Chaves personajes que no pertenecían a ella, confundiéndolos entre sí y originando los errores genealógicos presentes en la Nueva Corónica.

\section{La Primera Edad del Mundo}

La filiación de la primera edad da cuenta del plan inicial de Guaman Poma, cuyo objetivo era interrumpir la sucesión de Chaves cada ciertos patriarcas, con el objetivo de caracterizar estos personajes con la información del Anónimo (Tabla 2). Dado que el primer patriarca que menciona el Anónimo es Caín, el cronista interrumpió la sucesión de Chaves en Caynam asociando ambos nombres a un mismo personaje, cuando en realidad se trata de miembros de genealogías diferentes. Caín es el hijo de Adán y Eva que asesinó a su hermano Abel, mientras que Caynam es uno de los descendientes de Set, el hijo de Adán y Eva del cual se originaba la línea de los 
Tabla 2. La primera edad del mundo.

The first age of the world.

Guaman Poma (2004 [1615/1616]):23
"Adán engendró a Seth, Seth engendró a Enos, Enos engendró
a Caynam.
Caym mató a Abel;
déste salió la casta de los negros
por enbidia. Edeficó la primera ciudad; llamóla Enoch porque
un hijo suyo se llamaba ací
Caynam engendró a Malaleel, Malaleel engendró a Lareth,
Lareth engendró a Enoth,
Lareth engendró a Enoth,

el que está en el paraýso. Su padre deste, a Amelh, fue de linage de Caýn. Tubo tres hijos y una hija: Jael enuentó las cauanas, otro hijo, Tubal, enuentó el órgano y la uigüela y canto de órgano. Tabalcaym enbentó el arte de labrar hierro, la hija, Noema, enuentó el hilar.

Enochen engendró a Matuzalem.

Este dicho Matuzalem beuió en el mundo más que todos, mil y quarenta y tantos años,.

pero más ueuió Adán y Eua

Matusalén engendró a Lamech, Lamech engendró a Noé

Estos dichos hombres, cada uno de ellos y sus multiplicos, beuieron muy muchos años. Sólo Adán y Eua ueuiría dos o tres mil años. Parerían de dos en dos y ancí fue nesesario henchir el mundo de gente. Questo se escriue consederándose que no se puede sauer tanto ni de tantos años, porque el mundo está ya uiejo, que sólo Dios en su secreto saue todo lo pasado y lo uenedero. No puede pazar con lo determinado de Dios, cino sólo ymaginar que a millones de años desde que se fundó el mundo, según para el castigo de Dios. Estubo el mundo lleno de hombres que no cauía y estos no conoció al Criador y Hazedor de los hombres. Y ací, desto mandó Dios fuese castigado el mundo; dentro, todo criado, por sus pecados, fue castigado con las aguas del [di]luuio. Y fue determinado por Dios que se saluase Noé con sus hijos en la arca de Dios".
"Adam engendro a Seth. Seth engendro a Enos. Enos engendro a Caynam" (Chaves 1584 [1548]:57v)

“Caym mato a Abel ... (Anónimo 1554:71r)

Reflexión de Guaman Poma

... por embidia. Caym hedifico la primera ciudad, y llamo la Enoch, porque vn hijo suyo se llamaua assi” (Anónimo 1554:71r)

"Caynam vuo a Malaleel. Malaleel vuo a Iareth. Iaret vuo a Enoch" (Chaves 1584 [1548]:57v)

Enoch el que esta en parayso terrenal fue padre deste. Lamech fue del linaje de Caym, y fue el primer bigamo que se caso con dos mugeres. Tuuo tres hijos y vna hija. El vno llamo Jael, inuento las cauañas. El segundo llamado Thubal, inuento el organo, y la vihuela, y el canto. El tercero llamado Thubalcaym, inuento el arte de labrar hierro. La hija llamada Noema, inuento el hilar" (Anónimo 1554:71r)

Enoch vuo a Matusalem (Chaves 1584 [1548]:57v)

"En esta edad biuian los hombres mas de dcccc. años. Mathusalem biuio mas que todos, y biuio deccc. y. lcix. años. (Anónimo 1554:71r)

Reflexión de Guaman Poma

Matusalem vuo a Lamech. Lamech vuo a Noe. (Chaves 1584 [1548]:57v)

Reflexión de Guaman Poma ancestros de Jesús. Fuera de que ambas eran opuestas en virtud, la descendencia de Caín había perecido por completo tras el Diluvio Universal, de modo que su presencia en los cálculos cronológicos del Anno Mundi no tenía ningún sentido. El resultado de la confusión fue que Guaman Poma se desvió hacia la genealogía "mala" de Caín, cuestión que se complejizó aún más cuando el cronista identificó a Caynam-Caín con el origen de la casta de los negros, que era atribuido por la tradición medieval a Cam, el hijo de Noé que tras 
ver desnudo y borracho a su padre fue maldecido por éste (Gn 9:18-28). La asociación entre Cam y la casta de los esclavos también está presente en el Tercero Cathecismo y con un error similar al de Guaman Poma, ya que si bien en el sermón XXII -que trata justamente de cómo se han de honrar y obedecer los padres carnales- señala que "Sen y Iafet, dize la palabra de Dios que fueron buenos hijos y honrraron a Noe su padre. Can, que era otro hijo le deshonro y por esso el mal hijo, quedo hecho esclauo de los otros dos buenos hijos" (1585:132v), en la tabla de materias dicho episodio aparece referido como "Cain fue maldito y porque". Es más que probable que esta circunstancia y el parecido de los nombres -Caynam, Caín y Cam-haya confundido a Guaman Poma. Por otra parte, el tema de los negros y su origen debió ser de mucho interés para el cronista, que dedicó un capítulo completo de la Nueva Corónica a los negros africanos (Guaman Poma 2004 [1615/1616]:717725), cuya nación ocupaba un lugar específico en el nuevo y estricto orden jerarquizado que el cronista propuso a Felipe III (Ossio 1973:180).

Una segunda confusión en la redacción de la primera edad del mundo se originó a partir de un error similar al anterior, cuando Guaman Poma nuevamente interrumpió la sucesión de Chaves para volver al Anónimo en Enoc -hijo de Jared y por ende miembro de la línea de Set- que los comentaristas de la Biblia situaban en el paraíso porque había caminado con Dios (Gn 5:24). El problema es que en el Génesis existen dos personajes llamados Enoc: el ya señalado y el hijo de Caín, cuyo nombre había sido dado a la primera ciudad fundada fuera del paraíso (Gn 4:17). El Anónimo menciona a ambos, sin hacer del todo explícita la diferencia entre uno y otro, cuestión que debió despistar a Guaman Poma y que terminó por desviarlo nuevamente de la "línea buena" de Set que seguía Chaves. Eso explica la mención a Lamec, el primer bígamo de la historia según la tradición medieval, y a sus cuatro hijos Jabal, Jubal, Tubal-caín y Naama (Gn 4:19-22). La bigamia y otros pecados análogos fueron condenados por el cronista en reiteradas ocasiones a lo largo de la Nueva Corónica, lo que explica que la referencia a la bigamia de Lamec haya sido la única línea de este trozo omitida por el cronista en el proceso de copia.

\section{La Segunda Edad del Mundo}

Guaman Poma inaugura la descripción de la segunda edad (Tabla 3) con un cálculo cronológico de su propia pluma -6.612 años-que obtuvo añadiendo 5.000 años a los 1.612 años que habían transcurrido desde el nacimiento de Cristo hasta su propio presente. Este cálculo de la edad del mundo, tal como han señalado diferentes investigadores (Adorno 2001; Ossio 1973; Wachtel 1976), constituye el marco cronológico sobre el cual el cronista ordenó su narración y aparece reiteradamente a lo largo de la Nueva Corónica, con algunas variaciones. Luego del cómputo, el autor continúa indicando que la duración del diluvio fue de cuarenta días y cuarenta noches, tal como aparece en el relato bíblico (Gn 7:4).

Sin embargo, en la frase siguiente Guaman Poma se aleja de la versión del diluvio transmitida por el Génesis, haciendo del profeta Elías un testigo de la gran inundación. De acuerdo al Antiguo Testamento, este profeta vivió muchos siglos después del diluvio de Noé, durante el reinado de Acab en Israel. La historia de Elías está contenida en el Libro de los Reyes y en uno de sus episodios narra cómo éste predijo una gran sequía en la región de Samaria, que Jehová envió al pueblo de Israel en castigo por adorar falsos ídolos y no respetar los mandamientos del único Dios. La historia continúa con los prodigios que Elías realizó frente a los sacerdotes de Baal, cuya impostura quedó en evidencia ante toda Samaria. Pero Elías no conforme con demostrar que eran falsos profetas, mandó a degollar a cada uno de estos sacerdotes, a continuación de lo cual hizo llover sobre Samaria. Los actos de Elías enfurecieron al rey Acab y lo obligaron a refugiarse en el desierto, por el cual caminó durante cuarenta días y cuarenta noches, hasta detenerse en el monte Horeb en donde Jehová se le apareció no en el fuerte viento, ni en el terremoto, ni en el fuego, sino en un silbo de aire apacible y suave (I R:19).

La figura de Elías luchando contra los falsos ídolos -esta vez a propósito del hijo del rey Acab llamado Ocozías- fue recogida por el Tercero Cathecismo, que en su sermón sobre las guacas y los hechiceros homologó los falsos ídolos del Antiguo Testamento a las guacas andinas 5 . Aunque este sermón no recoge la mención al aire delgado ni al terremoto, es posible que Guaman Poma haya leído esta historia en el Libro de la Oración y la Meditación de Fray Luis de Granada, que utilizó el relato de Elías en el monte Horeb para recomendar a los católicos que tuviesen sus corazones quietos y templados, como el aire delgado y manso a través del cual Dios se le había manifestado al profeta $^{6}$. Esto no es extraño si se considera que 
Tabla 3. La segunda edad del mundo.

The second age of the world.

Guaman Poma (2004 [1615/1616]:25)

"Desde el arca de Noé, del [di]luuio, says mil y seycientos doze años: Mandó Dios llouer quarenta días y quarenta noches, aún pesó cin escanpar y esto uastó para anegar el mundo.

Primero cintió Helías el estruyendo y el tenblor de la tierra y el toruellino que trastornaua los montes. Y después desta tenpestad, ciguióse aquel ayre delgado en que uenía Dios y sesaua el castigo de Dios y ancí quedó castigado el mundo por mandado de Dios.

Noé salió del arca y plantó uiña y de ello hizo uino y biuió del dicho uino y se enborrachó.

Y sus hijos ydeficaron la torre de Babelonia; por mandado de Dios tubieron de $[\mathrm{sic}]$ deferentes lenguages que antes tubieron una lengua. En esta edad uiuieron los hombres quatrocientos o trecientos años.

Mandó Dios salir desta tierra, derramar y multiplicar por todo el mundo. De los hijos de Noé, destos dichos hijos de Noé, uno de ellos trajo Dios a las Yndias; otros dizen que salió del mismo Adán. Multiplicaron los dicho[s] yndios, que todo lo saue Dios y, como poderoso, lo puede tener aparte esta gente de yndios.

En tienpo deluuio, como dicho es, engendró Noé a Arphaxad, Arphaxad engendró a Sali, Sali engendró a Heber, Heber engendró a Phalig, Phalig engendró a Reu, Reu engendró a Sarug, Sarug engendró a Nachor, Nachor engendró a Tharé, Tharé engendró a Abrahán.

Salió de la tierra de los caldeos y destroyeron a los dichos sodomestas, que comensó los primeros ýdolos del rrey niño $(\mathrm{sic})^{1}$. Y se comensó la dicha moneda. Abrahán y su linage comensaron a serconcidarse. Y sacrificó a Dios con su hijo".

1 La transcripción "rrey niño" no es correcta. Léase "rey Nino".

el Libro de la Oración fue una de las fuentes directas del Symbolo Indiano de Oré, con quien Guaman Poma compartió la admiración por Luis de Granada (Adorno 1978:155). El cronista también siguió la obra de Granada -específicamente el Memorial de la Vida Cristiana- en otros capítulos de la Nueva Corónica asociados a profetas del Antiguo Testamento (Adorno anotando a Guaman Poma 2004 [1615/1616]:109) pero, hasta donde sabemos, ni en Oré ni en Granada la figura de Elías está vinculada al Diluvio Universal como sucede en la Nueva Corónica. De algún modo,
Filiación

Reflexión de Guaman Poma
"La segunda edad tuuo principio desque Noe salio del arca. Noe planto viña y beuio del vino, y se emborracho. En esta edad hedificaron los hijos de Adam la torre de Babylonia, y tuuieron origen las lxxii. lenguas, que antes no auia mas de vna lengua, y biuian los hombres. ccc. cccc. años"

(Anónimo 1554:71r)

Reflexión de Guaman Poma

"Dos años despues del diluuio engendro Sem a Arphaxad. Arphaxat vuo a Sale. Sale engendro a Heber. Heber engendro a Phaleg. Phaleg engendro a Reu. Reu engendro a Sarug. Sarug engendro a Nachor. Nachor engendro a Thare. Thare engendro a Abraham" (Chaves 1584 [1548]:58)

"En esta edad mando Dios salir a Abraam dela tierra de los Caldeos, y destruyr a los Sodomitas, y començaron los ydolos por el rey Nino, y tuuo principio el Cuño dela moneda por Thare padre de Abraam, y por Nino. Semiramis madre de Nino rehedifico a Babylonia. En esta edad mando Dios a Abraam que se circuncidase el y toda su generacion, duro esta edad desde Noe hasta que Abraam fue a sacrificar a su hijo" (Anónimo 1554:71r)
Guaman Poma debió relacionar los elementos comunes en ambos episodios -la mención a cuarenta días y cuarenta noches, la lluvia y el castigo de Dios- fundiéndolos en una sola historia en la que el mundo quedó castigado no por la corrupción de los hijos de Caín, sino por la adoración de los falsos ídolos que Elías -convertido en un extirpador de idolatrías al estilo de Cristóbal de Albornoz- había combatido.

Luego de la asociación entre el Diluvio y Elías, Guaman Poma continuó el relato tomando del Repertorio anónimo la historia de la embriaguez 
de Noé y la de la Torre de Babilonia. Después de introducir una reflexión acerca de la multiplicación de los hijos de Noé, el cronista copió del texto de Chaves la genealogía desde Noé hasta Abraham sin interrupciones, para concluir la segunda edad con un último párrafo adaptado nuevamente del Anónimo.

\section{La Tercera Edad del Mundo}

Como se observa en la Tabla 4, Guaman Poma a partir de este apartado comenzó a resumir la información del Anónimo, omitiendo varias frases que debieron parecerle impropias como, por ejemplo, que Abraham engendró a Ismael en su esclava Agar o que David pecó con Betsabé. Guaman Poma fue un acérrimo crítico del mestizaje y del adulterio, de modo que no resulta extraño imaginar por qué no quiso incluir en su relato historias como estas. De hecho, en otra ocasión el cronista condena manifiestamente "el adulterio de David", lo que hace pensar que sus escrupulosas omisiones fueron conscientes (Guaman Poma 2004 [1615/1616]:109). Pero estas censuras introducidas por el propio Guaman Poma le jugaron una mala pasada porque al omitir la frase que hacía referencia a la esclava Agar, Ismael pasó a la Nueva Corónica como el progenitor de Isaac,

Tabla 4. La tercera edad del mundo.

The third age of the world.

Terzera edad del mundo, desde Abrahán:

Engendró a Ysmael da donde desendió los dichos moros. Ysmael engendró Ysac; se casó con Rebeca y parió Ysaú y Jacop. De Jacop multiplicó Juzep.

Muyzén zacó a los hijos de Ysrrael del cautiuerio de Ygipto quando fue los dies plagas por mandado de Dios. Samoel profeta, fue Saúl. De Saúl fue rrey

Dauid, conpuso el pesalterio. Apzalón, su hijo. Salomón conpuso los Egleciásticos, edeficó tenplos a Dios. Daquí salió Juachín y Santa Ana, madre de Nuestra Señora Santa María, da donde nació Nuestro Señor Jesucristo,

saluador del mundo, hijo de Dios bibo. Es la casta y linage y desendencia de Jesucristo. Bolbiendo arriua a la generación de Abrahán:

Engendró Ysac, Ysac engendró a Jacop, Jacop fue a Egipto. Engendró a Jugep, ciendo 92 (sic) ${ }^{1}$ años. Estubo el pueblo en Egipto; engendró a Muyzén. Gouernó Jusué, fue capitán, Otonel fue jues, estubieron cin jues. Aod rregió el pueblo, Sangar fue jues, estubieron cin jues, Débora y Barach, estubieron cin jues. Gedeón gouernó, Abemelech fue jues, estubieron cin jues. Tola gouernó, Jaer fue jues, unos años estubieron cin jues. Lepre fue jues, estubieron cin jues. Abelsan fue jues, Elón fue jues, Apdón fue jues, estubieron cin jues. Sansón fue jues, estubieron cin jues, Hilí saserdote, Zamoel profeta, Saúl con Zamoel, la terzera edad del mundo, rrey Dauid.
"La tercera edad tuuo principio quando Abraam fue a sacrificar a Isaac. Abraam engendro a Hismael de su esclaua Agar. Deste Hismael descienden los moros. A Isaac caso su padre con Rebeca, y pario de vn vientre a Esau y Jacob /.../ Jacob tuuo dos mugeres hermanas, a Lia la legañosa, y a Rachel la hermosa y dos concubinas, a Celpha y a Bala /.../ Joseph su onzeno hijo fue vendido por sus hermanos /.../

La quarta edad tuuo principio en Moysen. Este Moysen saco a los hijos de Israel del captuerio de Egipto por mandando de Dios. En su tiempo fueron las diez plagas de Egypto /.../ Fue Samuel propheta, y Saul primero rey de Israel /.../

La quinta edad tuuo principio en el rey Dauid. Este Dauid fue sanctissimo, compuso el psalterio, mato ochocientos philisteos de vn encuentro, pecco con Bersabe, y mando matar a su marido Urias, su hijo Absalon lo echo del reyno. Hijo deste fue Salomon. Salomon compuso los ecclesiastes y los prouerbios, y los cantares, y hedifico el templo /.../Y fue sancta Ana, y sant Joachin, y nascio nuestra señora, y fue presentada en el templo donde encarno el hijo de Dios" (Anónimo 1554:71-72)

\section{Reflexión de Guaman Poma}

"Abraham vuo a Isaac. Isaac vuo a Iacob. Iacob vino a Egipto y vuo a Ioseph siendo de 91 años. Estuuo el pueblo en Egypto. Moysés gouerno. Iosue fue capitan. Othoniel fue juez. Estuuieron sin juez. Aod rigio el pueblo. Sangar fue juez. Estuuieron sin juez. Debora y Barach. Estuuieron sin juez. Gedeon gouerno. Abimelech fue juez. Tola gouerno. Iayr fue juez años. Estuuieron sin juez. Iepte fue juez. Abessan fue juez. Elon fue juez. Abdon fue juez. Estuuieron sin juez. Sanson fue juez. Estuuieron sin juez. Hely sacerdote. Samuel propheta. Saul con Samuel" (Chaves 1584 [1548]:61-61v).

1 La transcripción "92" no es correcta. Léase "91". 
y no como su hermanastro, como en efecto lo era. Por otro lado, al continuar su resumen del Anónimo, Guaman Poma también obvió que en éste la tercera edad concluía con Moisés, la cuarta con David y la quinta con el nacimiento de Jesús, condensando toda la información correspondiente a estos períodos en unas pocas líneas, sin distinguir cuándo comenzaba o terminaba una determinada edad. Esto explica la aparente incoherencia en la descripción de la tercera edad de la Nueva Corónica, en la que el cronista menciona a Moisés y otros patriarcas que en su propia periodificación pertenecen a las edades posteriores, como David y Salomón. No obstante, lo más importante es que después de condensar toda esta información, Guaman Poma abandonó por completo el Repertorio anónimo, utilizando en la redacción de las edades posteriores sólo las tablas cronológicas de Hieronimo de Chaves y sus propias reflexiones.

\section{La Cuarta Edad del Mundo}

Este apartado comienza con la genealogía desde el rey David hasta Sedequías que Guaman Poma (2004 [1615/1616]:29) copió textualmente del texto de Chaves (1584 [1548]:62), a continuación de la cual incluyó un comentario en el que indica que durante esta época se alzaron muchos reyes y señores. También señaló que en este tiempo se comenzó a vivir en policía por gobierno y por beneficio, mencionando de paso que procuraron tener mucha hacienda y riquezas. Esta descripción coincide con las características que el propio Guaman Poma atribuyó a la cuarta edad de los indios o Auca runa, que corresponde al período paralelo a la cuarta edad del mundo en la serie de las edades andinas. Este período también es caracterizado por el cronista como una época en que "comensaron a rreñir y batalla y mucha guerra y mortanza con su señor y rrey y con otro señor y rrey /.../ Y tenían mucho oro y plata", agregando que a raíz de las guerras los indios abandonaron la vida en los territorios bajos que llevaban hasta entonces para trasladarse a vivir a los pucaras bajo la protección de un señor (Guaman Poma 2004 [1615/1616]:64). De otra parte, a lo largo de la caracterización de la época de Auca Runa, Guaman Poma constantemente insiste en la idea de que para entonces los indios vivían bajo estrictas normas que castigaban el adulterio, la borrachera, el robo y la ociosidad, y que había mucha justicia (Guaman Poma 2004 [1615/1616]:64-78). Estos son todos elementos de una vida en policía, concepto que debió resultarle familiar al cronista después de la política reduccional llevada a cabo en los Andes por el virrey Toledo, a fines del siglo XVI. Aunque en el capítulo de las edades andinas Guaman Poma atribuye a Purun Runa -la tercera edad- los inicios de la vida en policía (Guaman Poma 2004 [1615/1616]:58), describiendo más adelante la policía y cristiandad de los indios del reino menciona explícitamente que en "Auca Runa comensó pulicía", a propósito de los trajes que los indios usaban antes del tiempo de la conquista hispana (Guaman Poma 2004 [1615/1616]:875). En consecuencia, Guaman Poma supuso que la cuarta edad del mundo y su equivalente en la serie andina se habían desarrollado simultáneamente y que, por ende, lo que había ocurrido en una necesariamente debía estar presente en la otra. El paralelismo que Guaman Poma supuso entre ambas series de edades ha sido propuesto a partir de la comparación de los dibujos que inauguran la primera edad del mundo y la primera de los indios (Duviols 1980:9; Plas 1996:111), en la medida en que ambos comparten muchas similitudes, en especial la forma en que Adán y el primer hombre andino aparecen representados. No obstante, existen otros indicios en la Nueva Corónica que sugieren que Guaman Poma percibió los eventos del pasado como universales, en el sentido de que habían tenido lugar a ambos lados del océano Atlántico. Uno de estos indicios es mencionado a raíz de la descripción de los castigos que Dios envió al inca Pachacuti por su idolatría, cuyo gobierno fue identificado por el cronista con pestilencias y sequías que asumió como mundiales: "en todo el mundo fue castigado en este tienpo y en Jerosalem los ciete años de hambre" (Guaman Poma 2004 [1615/1616]:109). Guaman Poma volvió sobre esta misma idea en el capítulo de los ídolos, suponiendo que los siete años de abundancia y los siete de hambruna que protagonizó José (Gn 41) -por cierto no en Jerusalén sino en Egipto- habían sido contemporáneos al gobierno de Pachacuti: "en tienpo de Pachacuti Ynga de los ciete años de hambre de Exipto" (Guaman Poma 2004 [1615/1616]:286).

\section{La Quinta Edad del Mundo}

La quinta edad del mundo arranca con dos comienzos, puesto que si bien Guaman Poma tituló la hoja correspondiente con el enunciado "Quinta edad del mundo, desde el nacimiento de Nuestro Señor y Saluador Jesucristo" (2004 [1615/1616]:31), 
la sucesión de reyes que copió de Chaves para el período comienza con el rey persa Ciro y no con el nacimiento de Jesús como lo anuncia el título (Chaves 1584 [1548]:63-63v). De hecho, en la Chronographia es la sexta edad la que comienza con el nacimiento de Jesús (Chaves 1584 [1548]:64), lo que indica que Guaman Poma nuevamente fusionó dos edades en una, algo parecido a lo que había hecho anteriormente con el Repertorio anónimo. No obstante la fusión, Guaman Poma mantuvo el orden de la sucesión tal como aparecía en Chaves, copiando los reyes persas y egipcios de la tabla cronológica de la quinta edad y luego el catálogo de emperadores romanos desde Julio César hasta Maximiliano I (Chaves 1584 [1548]:62v-64v). Cabe mencionar que las interpolaciones que Guaman Poma introdujo en estas series corresponden a pequeños párrafos que le sirvieron al autor para establecer nexos cronológicos entre las edades del mundo y las edades andinas indicando, por ejemplo, que Jesucristo había nacido en Belén cuando Cinche Roca tenía ochenta años en el Perú.

\section{Conclusiones}

Los errores en las genealogías de los patriarcas bíblicos presentes en la Nueva Corónica, lejos de constituir un simple descuido de Guaman Poma, evidencian su preocupación por tratar de elaborar una representación lineal y coherente del pasado occidental, utilizando como fuentes dos libros aparentemente muy similares entre sí: la Chronographia o Reportorio de los Tiempos de Hieronimo de Chaves y el Repertorio anónimo. No obstante, en el proceso de composición del apartado de las edades del mundo, el cronista tropezó con una serie de dificultades derivadas de las diferencias de contenido entre ambos registros, que no siempre fueron compatibles. Consciente de estas contradicciones, Guaman Poma paulatinamente fue abandonando el Repertorio anónimo, convirtiendo a la Chronographia en la única fuente textual de la cuarta y quinta edad. Sin embargo, al utilizar fuentes de tradición europea en la redacción de las edades del mundo es significativo recalcar que Guaman Poma no sólo actuó como un mero transmisor de la información y las periodizaciones presentes en ellas, sino que también intervino reelaborando estos contenidos, reflejando en este proceso la forma particular en que concibió la relación entre el pasado andino y el occidental. La más significativa de estas reelaboraciones fue la de ajustar la historia europea a un modelo temporal de cinco edades, comprimiendo los esquemas de seis edades que tanto Chaves como el Anónimo habían transmitido. Desde esta perspectiva, parece ser que Guaman Poma no se inspiró en un modelo europeo para determinar el número de las edades andinas, sino que más bien hizo todo lo contrario, acomodando las seis edades del mundo a una periodización de cinco etapas. La presencia de un modelo temporal análogo para el pasado andino está documentada en otras crónicas como las Memorias Antiguas Historiales y Políticas del Pirú de Fernando de Montesinos, que asignó una duración de mil años a cada una de las cinco edades o "soles", que a su vez se dividían en dos mitades de quinientos años cada una (Ossio 1973:189).

Este tipo de organización decimal estructurada sobre la base del número cinco también está presente en la periodización que el propio Guaman Poma estableció para la semana andina de diez días (2004 [1615/1616]:237,262), información que coincide con la transmitida por Blas Valera de acuerdo al registro del Inca Garcilaso (Adorno anotando a Guaman Poma 2004 [1615/1616]:58).

De otra parte, el rol fundacional del número cinco en otros contextos andinos también queda en evidencia en la organización del sistema administrativo incaico transmitida por el mismo Guaman Poma, que establecía un señor cada diez mil, mil y quinientos indios; un mandón cada cien, cincuenta y diez indios; y un mandoncillo cada cinco indios (Guaman Poma 2004 [1615/1616]:65). A este sistema también hace referencia Pedro Sarmiento de Gamboa en su Historia de los Incas (Sarmiento 1942 [1572]:147).

Finalmente, la construcción de secuencias ordinales y cardinales utilizando como criterio de clasificación el número cinco -o los conjuntos de cinco cosasconstituye un modelo de representación numérico profundamente arraigado tanto en las sociedades andinas prehispánicas como en las contemporáneas, tal como ha sido constatado por Gary Urton a través de la comparación de documentación etnohistórica con registros etnográficos actuales. Para Urton, los modelos de representación andinos basados en el número cinco descansan sobre un principio jerárquico en el cual el número "uno" o "primero" es asociado al origen de toda la serie, siendo identificado con una progenitora en edad madura (mama). El resto de las series que comprende desde el 2 al 5 se considera la descendencia del número uno, organizada 
gradualmente de acuerdo a su valor numérico. Para el autor, este principio de carácter quinario funcionaría combinado con otro de tipo dual, constituyendo en conjunto la base del sistema decimal de numeración presente en la cultura quechua. Este sistema queda en evidencia, por ejemplo, en la distribución de los diez ayllus de la comunidad de Pacaritambo en dos grupos de cinco, Hanansayaq y Hurinsayaq, siendo el ayllu de Nayhua perteneciente a la parcialidad de Hanansayaq reconocido como el primero y más antiguo de todos. La jerarquía implícita en el modelo de cincos también está presente en documentación colonial temprana como el Manuscrito de Huarochirí, en el cual sus protagonistas recurrentemente pertenecen a un grupo de cinco hermanos o el tiempo transcurrido se mide en períodos de cinco días (Urton 1997:78-80, 175-176, 214-216).

En conclusión, la correspondencia entre los ejemplos mencionados y el modelo de cinco edades del mundo presente en Guaman Poma sugiere que éste acomodó los esquemas europeos de representación del tiempo a una matriz andina, sincronizando ambos pasados y suponiendo que determinados eventos -como la vida en policía o la hambruna en tiempos de Pachacuti- habían sucedido simultáneamente tanto en el Viejo Mundo y como en las Indias.

Agradecimientos: Este artículo se realizó en el contexto del proyecto de investigación FONDECYT 1100132 "Historia de los Pueblos Andinos de Arica, Tarapacá y Atacama: Políticas Estatales y Eclesiásticas y las Dinámicas de las Comunidades Indígenas, siglos XVI-XIX”. Mis agradecimientos a Jorge Hidalgo por sus oportunas observaciones al borrador de este artículo; a Rolena Adorno, Mónica Barnes, David Fleming y Jean-Philippe Husson por la gentileza que tuvieron al enviarme sus artículos sobre Guaman Poma; a María Jesús Mancho y Alberto Blecua por sus orientaciones respecto al Repertorio anónimo; y a los evaluadores por sus sugerencias y correcciones.

\section{Referencias Citadas}

Acosta, J. y J. Atienza 1585. Tercero Cathecismo y Exposicion de la Doctrina Christiana. Impreso por Antonio Ricardo, Perú. http://www.archive.org/details/tercerocathecism01cath (8 marzo 2011).

Adorno, R. 1978. Las otras fuentes de Guaman Poma: sus lecturas castellanas. Revista Histórica 11(2):137-158.

- - - 2001. Guaman Poma y su crónica ilustrada del Perú colonial: un siglo de investigaciones hacia una nueva era de lectura. http://www2.kb.dk/elib/mss/poma/presentation/index. htm (12 marzo 2011).

Anónimo 1554. Repertorio de los Tiempos. Impreso en casa de Francisco Fernández de Córdova, Valladolid. http://gredos.usal. es/jspui/handle/10366/82575 (7 marzo 2011).

Barnes, M. 1995. Las edades del hombre y del mundo según Hierónimo Chaues, de Sevilla y Guaman Poma de Ayala, del Perú. En Humanismo Siglo XX. Estudios Dedicados al Dr. Juan Adolfo Vázquez, editado por J. Schobinger, pp. 291-297. Editorial Fundación Universidad Nacional de San Juan, San Juan.

Chaves, J. 1584 [1548]. Chronographia o Reportorio de los Tiempos. Impreso en casa de Fernando Díaz, Sevilla. http:// zaguan.unizar.es/documents/fondos/H_4_127.djvu (6 marzo 2011).

Durand, J. 1948. La biblioteca del Inca. Nueva Revista de Filología Hispánica II(3):239-264.

Duviols, P. 1980. Periodización y política: la historia prehispánica del Perú según Guaman Poma de Ayala. Boletín Instituto Francés de Estudios Andinos IX (3-4):1-18.

- - - 1983. Guaman Poma, historiador del Perú antiguo: una nueva pista. Revista Andina 1:103-115.
Fleming, D. 1994. Guaman Poma, Hieronymo de Chaves and the Kings of Persia. Latin American Indian Literatures Journal 1:46-60.

Granada, L. 1994 [1554]. Libro de la oración y meditación. En Obras Completas de Fray Luis de Granada I. Edición de Alvaro Huerga. Fundación Universitaria Española, Dominicos de Andalucía, Madrid.

Guaman Poma de Ayala, F. 2004 [1615/1616]. El Primer Nueva Corónica y Buen Gobierno. Transcripción y edición de John V. Murra, Rolena Adorno y Jorge L. Urioste, corregida por Ivan Boserup y Rolena Adorno. Edición virtual de Det Kongelige Bibliotek, Dinamarca. http://www.kb.dk/permalink/2006/poma/ titlepage/es/text/?open=id3083608 (7 marzo 2011).

Hampe, T. 1996a. Bibliotecas Privadas en el Mundo Colonial. La Difusión de Libros e Ideas en el Virreinato del Perú (siglos XVI y XVII). Vervuert-Frankfurt. Iberoamericana-Madrid.

- - - 1996b. Cultura Barroca y Extirpación de Idolatrías. La Biblioteca de Francisco de Avila - 1648. Centro de Estudios Regionales Andinos Bartolomé de las Casas, Cusco.

Husson, J. 1995. En busca de las fuentes indígenas de Waman Puma de Ayala. Las raíces incas y Yaruwillka del cronista indio: ¿invención o realidad? Revista Histórica 19:29-71.

Ossio, J. 1973. Guaman Poma: Nueva Coronica o carta al rey. Un intento de aproximación a las categorías del pensamiento del mundo andino. En Ideología Mesiánica en el Mundo Andino, editado por J. Ossio, pp. 155-213. Editorial de Ignacio Prado Pastor, Lima. 
Plas, S. 1996. Une source européenne de la Nueva Corónica y Buen Gobierno de Guaman Poma. Journal de la Société des Américanistes 82:97-116.

Real Academia Española. Corpus diacrónico del español. Banco de datos CORDE. http://www.rae.es (8 marzo 2011).

Sarmiento de Gamboa, P. 1942 [1572]. Historia de los Incas. EMECÉ Editores, Buenos Aires.
Urton, G. 1997. The Social Life of Numbers. University of Texas Press, Austin.

Wachtel, N. 1976 [1971]. Los Vencidos. Los Indios del Perú frente a la Conquista Española (1530-1570). Traducido por A. Escohotado. Alianza Editorial, Madrid.

\section{Notas}

1 Hasta donde sabemos, no existe una edición crítica de la Chronographia que dé cuenta de las variantes entre las diferentes ediciones. Para el presente artículo se ha tenido como texto base la edición de Sevilla de 1584, pero también se han consultado otras tres ediciones (Sevilla, 1566; Sevilla, 1576; Sevilla, 1580) digitalizadas por diferentes bibliotecas españolas. Las diferencias entre ellas se deben principalmente a correcciones -sobre todo en el capítulo del calendario con los días del año y las festividades de los santos- y adiciones realizadas por su propio autor. Comparando la forma y composición de los títulos en la Nueva Corónica y en la Chronographia, Plas (1996:102) sugiere que Guaman Poma pudo haber seguido una de las ediciones sevillanas de la casa de Fernando Díaz (1580, 1581, 1584).

2 La filiación entre este libro y la Nueva Corónica se realizó intentando múltiples entradas a través del CORDE.

3 La Chronographia o Reportorio de los Tiempos de Hieronimo de Chaves y el Repertorio de los Tiempos anónimo tienen en común el prólogo al prudente y sabio lector, así como también el capítulo de las divisiones de los días, meses y años, el de la clasificación de las regiones del cielo y el del calendario de los días del año y las festividades de los santos. Dado que este último capítulo es el que más variantes presenta en las diferentes ediciones de la Chronographia y que fue copiado tanto por Guaman Poma como por el
Anónimo, un cotejo entre todas sus versiones ayudaría a determinar, de un lado, qué edición de Chaves siguió el Repertorio anónimo y, de otro, cuál siguió Guaman Poma. 4 Los capítulos de Chaves omitidos por el Repertorio anónimo son, entre otros, el de las particularidades del año lunar, embolismal, discreto, platónico y sus respectivas indicciones; los meses peragratorios y los días idus; la división del mundo y sus partes; $y$ las epactas de la luna y los intervalos de las fiestas movibles. En cualquier caso, la comparación que hemos realizado entre la Chronographia y el Repertorio anónimo ha sido superficial, principalmente porque no constituye el tema central del presente estudio. Futuras investigaciones podrán aportar una filiación mucho más completa de la que hemos ofrecido aquí.

5 "Sabeys que quenta la Sagrada Escriptura, que un Rey llamado Ocozias embio a consultar la guaca, estando enfermo si sanaria, y enojado Dios, le embio a dezir con su Propheta Elias, que porque auia consultado a Belzebud que era guaca, y no auia consultado, ni llamado a Dios, por esso moriria, y no sanaria de aquel mal" (Tercero Cathecismo 1585:100).

6 "Y por esta misma causa, cuando apareció a Elías en el monte, no le apareció en la tempestad, ni en el terremoto, ni en el fuego, sino en aquel silbo de aire delgado y blando, que es el corazón pacífico y reposado el cual es templo vivo y morada de Dios" (Granada 1994 [1554]:308-309). 